\title{
A Visual Comparison of Gaze Behavior from Pedestrians and Cyclists
}

\author{
Mathias Trefzger \\ Karlsruhe University \\ of Applied Sciences \\ mathias.trefzger@ \\ outlook.com
}

\author{
Tanja Blascheck \\ Inria Saclay \\ tanja.blascheck@ \\ inria.fr
}

\author{
Michael Raschke \\ Blickshift GmbH \\ michael.raschke@ \\ blickshift.de
}

\author{
Sarah Hausmann \\ Karlsruhe University \\ of Applied Sciences \\ sarah.hausmann@ \\ hs-karlsruhe.de
}

\author{
Thomas Schlegel \\ University of Applied \\ Sciences Karlsruhe \\ thomas.schlegel@ \\ hs-karlsruhe.de
}

\begin{abstract}
In this paper, we contribute an eye tracking study conducted with pedestrians and cyclists. We apply a visual analytics-based method to inspect pedestrians' and cyclists' gaze behavior as well as video recordings and accelerometer data. This method using multi-modal data allows us to explore patterns and extract common eye movement strategies. Our results are that participants paid most attention to the path itself; advertisements do not distract participants; participants focus more on pedestrians than on cyclists; pedestrians perform more shoulder checks than cyclists do; and we extracted common gaze sequences. Such an experiment in a real-world traffic environment allows us to understand realistic behavior of pedestrians and cyclists better.
\end{abstract}

\section{CCS CONCEPTS}

- Human-centered computing $\rightarrow$ Visual analytics; Empirical studies in ubiquitous and mobile computing;

\section{KEYWORDS}

eye tracking, pedestrians, cyclists, visual analytics, real-world environment, traffic situations, multi-model data

ACM Reference Format:

Mathias Trefzger, Tanja Blascheck, Michael Raschke, Sarah Hausmann, and Thomas Schlegel. 2018. A Visual Comparison of Gaze Behavior from Pedestrians and Cyclists. In ETRA '18: 2018 Symposium on Eye Tracking Research and Applications, fune 14-17, 2018, Warsaw, Poland. ACM, New York, NY, USA, 5 pages. https://doi.org/10.1145/3204493.3204553

\section{INTRODUCTION}

In recent years, the number of injured pedestrians in Germany has increased from 33.499 in 2013 to 34.050 in 2016 and the number of injured cyclists has risen from 77.439 in 2013 to 87.992 in $2016^{1}$. Most of the registered accidents, during which people were

\footnotetext{
${ }^{1}$ https://www.destatis.de/

Permission to make digital or hard copies of all or part of this work for personal or classroom use is granted without fee provided that copies are not made or distributed for profit or commercial advantage and that copies bear this notice and the full citation on the first page. Copyrights for components of this work owned by others than the author(s) must be honored. Abstracting with credit is permitted. To copy otherwise, or republish, to post on servers or to redistribute to lists, requires prior specific permission and/or a fee. Request permissions from permissions@acm.org.

ETRA '18, June 14-17, 2018, Warsaw, Poland

(C) 2018 Copyright held by the owner/author(s). Publication rights licensed to the Association for Computing Machinery.

ACM ISBN 978-1-4503-5706-7/18/06 ..\$15.00

https://doi.org/10.1145/3204493.3204553
}

injured, happened in urban regions and within city velocity limits. Therefore, pedestrians and cyclists are often involved in accidents.

Eye tracking experiments support to analyze if drivers, cyclists, and pedestrians are aware of each other. Whereas researchers have analyzed the visual perception of car drivers in many eye tracking experiments [Kapitaniak et al. 2015], there are only few experiments performed with pedestrians and cyclists. In recent years, researchers have published a growing number of eye tracking experiments analyzing pedestrians and cyclists (e. g., [Davoudian and Raynham 2012; Kiefer et al. 2012; Mantuano et al. 2016; Vansteenkiste et al. 2014; Wenczel et al. 2017]). In this paper, we apply a visual analyticsbased method to analyze gaze behavior of pedestrians and cyclists in a real-world eye tracking experiment.

\section{RELATED WORK}

The use of mobile eye tracking solutions for analyzing pedestrians and cyclists has been steadily increasing. In the beginning, researchers mainly used mobile eye trackers in controlled settings, for example, in artificial laboratories simulating a traffic environment [Bock et al. 2015; Igari et al. 2008; Jäger et al. 2015; Marigold et al. 2007; Zito et al. 2015] or performing experiments indoors [Jovancevic-Misic and Hayhoe 2009; Schnitzler et al. 2016; Vansteenkiste et al. 2013]. However, these eye tracking experiments do not reflect the realistic behavior and situation of pedestrians and cyclists in traffic environments. Therefore, researchers are now executing a growing number of experiments in real-world environments. Multiple eye tracking experiments exist that analyze pedestrians' [Brügger et al. 2018; Davoudian and Raynham 2012; Fotios et al. 2015a,b; Kiefer et al. 2014, 2012; Wenczel et al. 2017] or cyclists' behavior [Mantuano et al. 2016; Schmidt and von Stülpnagel 2018; Vansteenkiste et al. 2014]. In contrast to existing works, we are presenting a visual analytics-based approach to explore patterns, extract common eye movement strategies, and enable a combined analysis of the multi-modal data.

In our eye tracking study, we use a visual analysis method to analyze our data. Multiple examples for using a visual analytics approach are available in the eye tracking literature [Andrienko et al. 2012; Blascheck et al. 2016c; Kurzhals et al. 2014, 2016; Kurzhals and Weiskopf 2013; Raschke et al. 2014]. In their state-of-the-art report, Blascheck et al. [2017] have collected many different visualization techniques for eye movement data.

In addition, a combination of multiple data sources is beneficial. Previous work gives examples of visualization techniques incorporating multiple data sources for analyzing eye tracking data. For example, a combination of eye movement data and interaction logs [Crowe and Narayanan 2000]; eye movement data and 


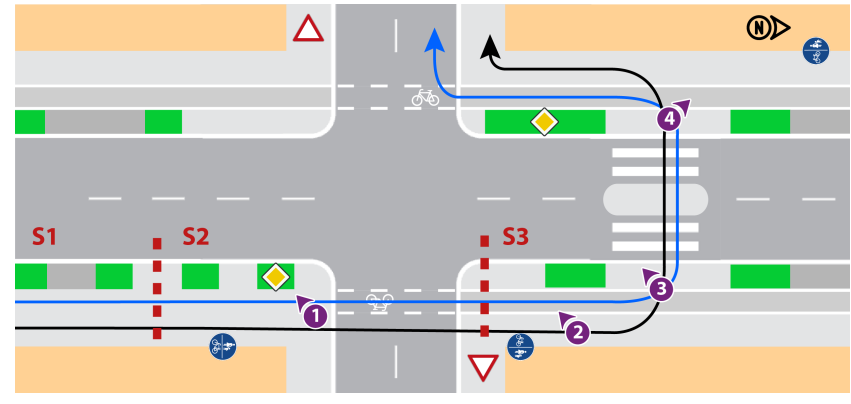

Figure 1: Segment of the route divided into three subsegments: 1) straight pavement where cycling is allowed, 2) non-traffic regulated crossing, and 3) crosswalk.

Map legend: bicycle lane (blue line); pedestrian lane (black line); parking lines (dark gray rectangles), grassed areas (green rectangles); houses (light orange rectangles); shoulder check positions (purple circles).

think-aloud protocols [Franchak et al. 2010]; eye movement data, video data, and paper notes [Fouse et al. 2011; Weibel et al. 2012]; or eye movement data, interaction logs, and think-aloud protocols [Blascheck et al. 2016a,b,c].

\section{VISUAL ANALYSIS OF PEDESTRIAN AND CYCLIST BEHAVIOR}

In the following section, we present an eye tracking experiment conducted in a real-world traffic environment with cyclists and pedestrians. We analyze the data using a visual method, which we shortly introduce. Then, we discuss exemplary results and experiences gained during the experiment.

\subsection{Experiment Design and Questions}

To investigate behavior of pedestrians and cyclists, we conducted an experiment, in which participants had to follow a predefined route in an urban area of a town in Germany. Participants had to cycle and walk the route.

With the collected data, we want to answer multiple questions:

- Which objects as well as obstacles are focused on most and how distracting are advertisements at the side of the road (Section 3.5.1)?

- What common gaze sequences do pedestrians and cyclists perform and how does their behavior differ (Section 3.5.2)?

- How long and when do pedestrians and cyclists perform shoulder checks, especially if entering a potentially dangerous traffic situation (Section 3.5.3)?

In this paper, we only analyze a part $(160 \mathrm{~m})$ of a longer route $(1.5 \mathrm{~km})$ participants had to cycle and walk. We sub-divided this part of the route into three sub-segments (cf. Figure 1).

\subsection{Participants}

We performed the experiment with 27 participants in total. However, due to difficulties with the eye tracking equipment, we were only able to use eye movement data with sufficient accuracy of 13 pedestrians ( 2 females; age: $M=27.8$ years \pm 8.86 ) and 14 cyclists

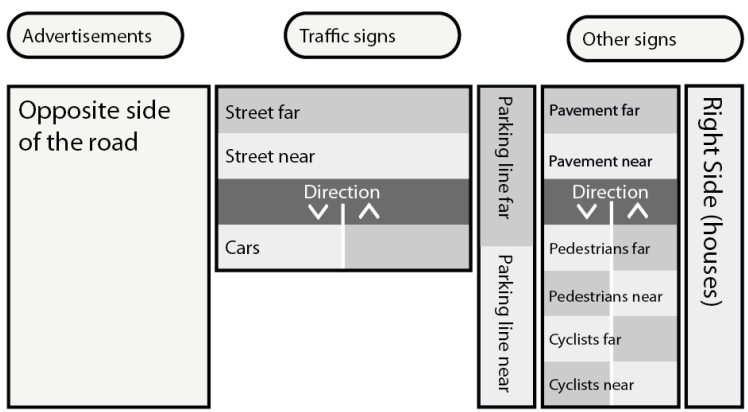

Figure 2: Example of the abstract reference image of the 21 AOIs for segment S1.

(3 females; age: $M=27.9 \pm 8.69$ ). All participants had normal or corrected-to-normal vision.

\subsection{Experimental Procedure}

We asked participants to walk or cycle the predefined route. We counter-balanced the order of participants walking or cycling to avoid learning effects when performing the route a second time. At the beginning of each trial, we asked participants to sign a consent form and fill out a demographic questionnaire. We used the Tobii Pro Glasses 2 to record the data and we performed the 1-point calibration procedure to calibrate the eye tracker. Afterwards, we checked the calibration by asking participants to focus on different points. We did the calibration indoors to ensure accuracy.

Because the goal of the experiment was to understand behavior of pedestrians and cyclists, we did not give participants a specific task. We only instructed them to walk or cycle the given route. However, we gave participants three instructions before they started their trip, to ensure a save travel within the traffic environment:

(1) By foot and while cycling the participants should cover the route on the right side of the street.

(2) While cycling the participants should only ride on paths allowed for cycling.

(3) Participants should exactly follow the given route.

\subsection{Multi-model Visual Analysis Approach}

We apply a visual analysis approach, which combines multi-modal data collected during our eye tracking experiment with pedestrians and cyclists. The applied method consists of the three steps:

(1) Data collection

(2) AOI Mapping

(3) Data Analysis

3.4.1 Data Collection. We recorded the eye movement data with the Tobii Pro Glasses 2 at $50 \mathrm{~Hz}$. The eye tracking glasses also record the accelerometer data and a video of the field of view. Participants wore the glasses, which feature a recording unit. We conducted the experiment outside during different weather conditions. Therefore, we had to exclude some data of participants with a precision lower than $80 \%$.

3.4.2 AOI Mapping. Because of the complex traffic environment, we use an abstract reference image of the scene to define AOIs and map fixations to them (cf. Figure 2). The eye movement data is 
Pedestrians
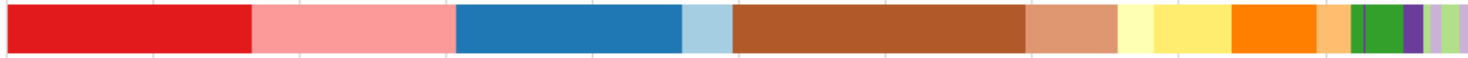

Cyclists
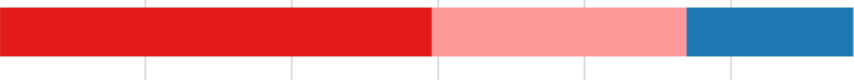

\begin{tabular}{|c|c|c|}
\hline $10 \%$ & $40 \%$ & $50 \%$ \\
\hline - Pavement close & = Pavement far & - Parkingslots close \\
\hline E Right side (houses) & Etreet & Advertisements and non-traffic signs \\
\hline Vehicle direction north & Wehicle direction south & - Pedestrian direction north far \\
\hline - Pedestrian direction south far & - Pedestrian direction south close & Cyclist direction north far \\
\hline
\end{tabular}

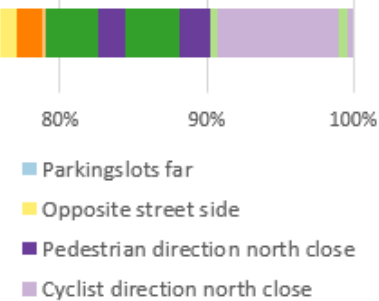

Eyclist direction south far

acyclist direction south close

Figure 3: AOI visit duration of pedestrians (top) and cyclists (bottom) for different AOIs for segment S2.1.

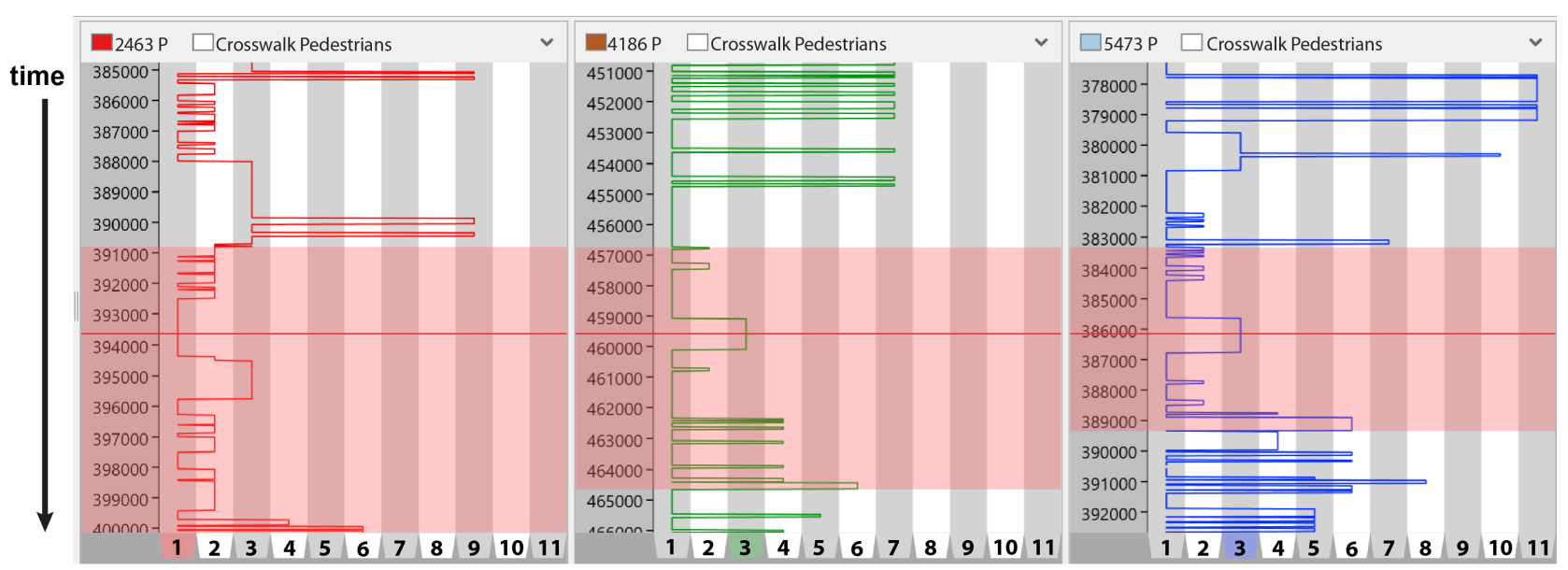

Areas of Interest
1. N/A
4. Pavement far
7. Right side (houses)
10. Opposite side of the road
2. Junction right
5. Pavement near
8. Street far
11. Junction right (road users)
3. Schoulder check left
6. Parking line
9. Street near

Figure 4: Parallel scanpath visualization showing the five AOI long gaze sequence junction right-shoulder check left - junction right - pavement far - parking line for three participants colored in red, green, and blue.

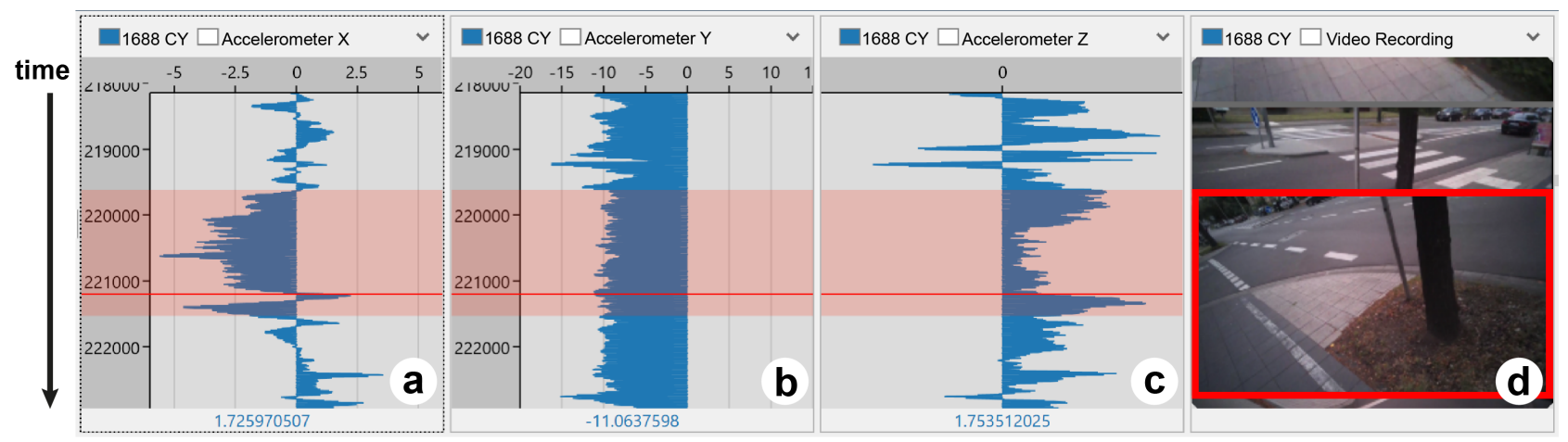

Figure 5: Line graph showing data of the accelerometer: a) $x$-direction, b) $y$-direction, which represents gravity, c) $z$-direction, and d) a frame from the video recording. The red highlight shows the time when the cyclist performed a shoulder check. 
mapped frame-by-frame onto the abstract reference image. We also define distractor AOIs [Devillez et al. 2017] to capture other road users (i. e., car drivers, cyclists, pedestrians) and the direction in which they were heading. This allows us to answer the question how other road users influence attention. In total, for each subsegment we defined AOIs: S1 has 21 AOIs (cf. Figure 2), S2 has 27 AOIs, and S3 has 16 AOIs.

3.4.3 Data Analysis. After mapping fixations to AOIs, the next step is an annotation of interesting situations and an explorative visual analysis. For annotating and analyzing the data, we use a parallel visualization of video recordings, eye movement and sensor data. Thereby, we can complement the different data streams if data points are missing or hard to interpret. This allows us to receive more detailed and accurate data. Additionally, we use the parallel scanpath visualization by Raschke et al. [2012; 2014] to search for common sequences in the eye movement data.

Annotation. In traffic, performing shoulder checks before switching lanes or turning is important to avoid accidents. Because such eye movements occur fast fixations are missing. Therefore, we semi-automatically annotate the instances when participants look backwards. Figure 5 shows a plot of the accelerometer data. Figure 5 (a) depicts the $x$-direction of the accelerometer sensor, (b) the $y$ direction (representing gravity), and (c) represents the $z$-direction. Figure 5 (d) shows the current frame of the video recording. A spike in these graphs indicates a rapid change of the acceleration. We can label each instance of a shoulder check, in which the $x$-direction is $\leq 0$ and the $z$-direction is $\geq 0$.

Visual Analysis. Additionally, we analyze common sequences of AOIs using the parallel scan path method [Raschke et al. 2012, 2014]. This parallel visualization of video recordings and eye movement data allows us to identify interesting sections for different traffic situations visually. Using a visual analytics-based pattern search algorithms based on the Levenshtein distance [Levenshtein 1966], we find common eye movement strategies, respectively common behavior of the participants.

\subsection{Analysis and Results}

In the following sections, we discuss different observations to show how to analyze the collected multi-modal data. Based on the questions in Section 3.1, we first investigate segment S1 (cf. Section 3.5.1) to understand which objects and obstacles are focused on most and how distracting advertisements are at the side of the road. Next, we analyze segment S2 (cf. Section 3.5.2) to find common gaze sequences of pedestrians and cyclists and investigate how their behavior differs. Last, we explore segment S3 (cf. Section 3.5.3) to understand how long and when pedestrians and cyclists perform shoulder checks.

3.5.1 Segment S1 - Focus on Obstacles. Our first question (cf. Section 3.1) is about understanding, which objects and obstacles participants focus on most. In addition, we explore how distracting advertisements are at the side of the road. Therefore, we analyze the eye movement data based on the AOIs defined in Figure 2 for segment S1. Figure 3 shows an overview of how much attention pedestrians and cyclists paid to individual AOIs. Most attention was paid to the path itself (red AOIs), for cyclists more than for pedestrians. Another observation is that both pedestrians and cyclists focus on pedestrians (dark green and purple AOIs) longer than on cyclists (light green and purple AOIs). Advertisements (light yellow AOI) attracted the attention of pedestrians as well as cyclists, however, only minimally.

3.5.2 Segment S2 - Gaze Sequences. With the parallel scan path technique [Raschke et al. 2012, 2014], we found the longest sequence with five AOIs in the eye movement data of the pedestrians: junction right - shoulder check left - junction right - pavement far - parking line. Figure 4 shows this sequence. It represents a specific gaze behavior, which we classify as a gaze sequence for safe traveling, because it increases the probability that participants perceived the situation in its entirety. We also found shorter common AOI sequences for pedestrians, such as pavement near - pavement far as well as pavement far - pavement near. For cyclists common AOI sequences were parking line - sidewalk near - parking line, pavement near - pavement far - pavement near, or pavement near advertisement - street (right).

3.5.3 Segment S3-Shoulder Checks. One goal of the experiment was to measure and analyze if participants performed shoulder checks at important positions on the road. To analyze this, we defined four positions in the segment (cf. Figure 1, purple circles). As described above, we manually annotated shoulder checks using the accelerometer data and video recording of participants.

Overall, cyclists perform less shoulder checks and the average duration of shoulder check times are shorter than for pedestrians. The only exception is shoulder check SC 3 before cyclists cross the crosswalk. Another observation is that cyclists did not perform shoulder check SC 2; however, this could also be, because here pedestrians had to cross the bicycle lane. While the missing shoulder checks are likely to have a negative impact on the safety, we believe the shorter shoulder checks of cyclists are a necessity, because of the higher speed, which leads to longer distances in blind flight that can be dangerous.

\section{CONCLUSION AND DISCUSSION}

In this paper, we analyzed and compared gaze behavior of pedestrians and cyclists using a visual analysis approach of multi-modal data. We collected eye movement and accelerometer data as well as a video recording to enable a holistic analysis of pedestrians and cyclists' behavior. When investigating eye movement data in real-world traffic situations, we had to define a high number of AOIs. Therefore, we use an abstract reference image for mapping eye movement data onto AOIs. For the analysis of the recorded multi-modal data, we use a visual approach for a detailed analysis and comparison of the data. The visual analysis showed that the explorative analysis with multiple data streams leads to an efficient overview of the collected data. We exemplary analyzed data from a real world eye tracking experiment. Our main results of the analysis are that participants paid most attention to the path itself; advertisements do not distract pedestrians and cyclists much; and participants focus more on pedestrians than on cyclists. We were also able to extract common gaze sequences, and we analyzed how often pedestrians and cyclists perform shoulder checks. 


\section{REFERENCES}

Gennady Andrienko, Natalia Andrienko, Michael Burch, and Daniel Weiskopf. 2012 Visual Analytics Methodology for Eye Movement Studies. IEEE Transactions on Visualization and Computer Graphics 18, 12 (2012), 2889-2898.

Tanja Blascheck, Fabian Beck, Sebastian Baltes, Thomas Ertl, and Daniel Weiskopf. 2016a. Visual Analysis and Coding of Data-Rich User Behavior. In Proceedings of the IEEE Conference on Visual Analytics Science and Technology. IEEE Computer Society Press, 141-150.

Tanja Blascheck, Markus John, Steffen Koch, Leonard Bruder, and Thomas Ertl. 2016b. Triangulating User Behavior Using Eye Movement, Interaction, and Think Aloud Data. In Proceedings of the Symposium on Eye Tracking Research \& Applications. ACM, 175-182.

Tanja Blascheck, Markus John, Kuno Kurzhals, Steffen Koch, and Thomas Ertl. 2016c. $V^{2}$ : A Visual Analytics Approach for Evaluating Visual Analytics Applications. IEEE Transactions on Visualization and Computer Graphics 22, 1 (2016), 61-70.

Tanja Blascheck, Kuno Kurzhals, Michael Raschke, Michael Burch, Daniel Weiskopf and Thomas Ertl. 2017. Visualization of Eye Tracking Data: A Taxonomy and Survey. Computer Graphics Forum 36, 8 (2017), 260-284.

Otmar Bock, Paolo Riccardo Brustio, and Steliana Borisova. 2015. Age-Related Differences of the Gaze Pattern in a Realistic Pedestrian Traffic Task. International Journal of Applied Psychology 5, 1 (2015), 13-19.

Annina Brügger, Kai-Florian Richter, and Sara Fabrikant. 2018. Which egocentric direction suffers from visual attention during aided wayfinding? In Eye Tracking for Spatial Research, Proceedings of the 3rd International Workshop. ETH Zurich, $22-27$.

Eric Crowe and Hari Narayanan. 2000. Comparing interfaces based on what users watch and do. In Proceedings of the Symposium on Eye Tracking Research \& Applications. ACM, 29-36.

N Davoudian and P Raynham. 2012. What do pedestrians look at at night? Lighting Research \& Technology 44, 4 (2012), 438-448.

Hélène Devillez, Anne Guérin-Dugué, and Nathalie Guyader. 2017. How a distractor influences fixations during the exploration of natural scenes. Fournal of Eye Movement Research 10, 2 (2017), 1-13.

Steve Fotios, Jim Uttley, and B Yang. 2015a. Using eye-tracking to identify pedestriansâĂŹ critical visual tasks, Part 1. Dual task approach. Lighting Research \& Technology 47, 2 (2015), 164-173.

Steve Fotios, Jim Uttley, and B Yang. 2015b. Using eye-tracking to identify pedestriansâĂŹ critical visual tasks, Part 2. Fixation on pedestrians. Lighting Research \& Technology 47, 2 (2015), 149-160.

Adam Fouse, Nadir Weibel, Edwin Hutchins, and James Hollan. 2011. ChronoViz: A system for supporting navigation of time-coded data. In CHI Extended Abstracts on Human Factors in Computing Systems. ACM, 299-304.

John Franchak, Kari Kretch, Kasey Soska, Jason Babcock, and Karen Adolph. 2010. Head-mounted eye-tracking of infants' natural interactions: A new method. In Proceedings of the Symposium on Eye Tracking Research \& Applications. ACM, 21-27.

D Igari, M Shimizu, and R Fukuda. 2008. Eye movements of elderly people while riding bicycles. In Proceedings of the 6th International Conference of the International Society for Gerontechnology. 1-4.

Michael Jäger, Thomas Nyffeler, R Müri, Urs Peter Mosimann, and Tobias Nef. 2015. Adapting a driving simulator to study pedestriansâĂŹ street-crossing decisions: a feasibility study. Assistive Technology 27, 1 (2015), 1-8.

Jelena Jovancevic-Misic and Mary Hayhoe. 2009. Adaptive gaze control in natural environments. Fournal of Neuroscience 29, 19 (2009), 6234-6238.

Bronislaw Kapitaniak, Marta Walczak, Marcin Kosobudzki, Zbigniew Jozwiak, and Alicja Borkiewicz. 2015. Application of eye-tracking in the testing of drivers: a review of research. International fournal of Occupational Medicine and Environmental Health 28, 6 (2015), 941-954.

Peter Kiefer, Ioannis Giannopoulos, and Martin Raubal. 2014. Where am I? Investigating map matching during self-localization with mobile eye tracking in an urban environment. Transactions in GIS 18, 5 (2014), 660-686.

Peter Kiefer, Florian Straub, and Martin Raubal. 2012. Towards Location-aware Mobile Eye Tracking. In Proceedings of the Symposium on Eye Tracking Research and Applications. ACM, 313-316.

Kuno Kurzhals, Florian Heimerl, and Daniel Weiskopf. 2014. ISeeCube: Visual Analysis of Gaze Data for Video. In Proceedings of the Symposium on Eye Tracking Research \& Applications. ACM, 43-50.

Kuno Kurzhals, Marcel Hlawatsch, Florian Heimerl, Michael Burch, Thomas Ertl, and Daniel Weiskopf. 2016. Gaze Stripes: Image-Based Visualization of Eye Tracking Data. IEEE Transactions on Visualization and Computer Graphics 22, 1 (2016), 10051014.

Kuno Kurzhals and Daniel Weiskopf. 2013. Space-Time visual analytics of eye-tracking data for dynamic stimuli. IEEE Transactions on Visualization and Computer Graphics 19, 12 (2013), 2129-2138.

Vladimir Levenshtein. 1966. Binary codes capable of correcting deletions, insertions and reversals. Soviet Physics-Doklady 10, 8 (1966), 565-572.

Alessandra Mantuano, Silvia Bernardi, and Federico Rupi. 2016. Cyclist gaze behavior in urban space: An eye-tracking experiment on the bicycle network of Bologna.
Case Studies on Transport Policy 5, 2 (2016), 408-416.

Daniel Marigold, Vivian Weerdesteyn, Aftab Patla, and Jacques Duysens. 2007. Keep looking ahead? Re-direction of visual fixation does not always occur during an unpredictable obstacle avoidance task. Experimental Brain Research 176, 1 (2007), $32-42$.

Michael Raschke, Xuemei Chen, and Thomas Ertl. 2012. Parallel scan-path visualization. In Proceedings of the Symposium on Eye Tracking Research \& Applications. ACM, 165-168.

Michael Raschke, Dominik Herr, Tanja Blascheck, Michael Burch, Michael Schrauf, Sven Willmann, and Thomas Ertl. 2014. A Visual Approach for Scan Path Comparison. In Proceedings of the Symposium on Eye Tracking Research \& Applications. ACM, 135-142.

Sonja Schmidt and Rul von Stülpnagel. 2018. Risk Perception and Gaze Behavior during Urban Cycling-A Field Study. In Eye Tracking for Spatial Research, Proceedings of the 3rd International Workshop. ETH Zurich, 34-39.

Verena Schnitzler, Ioannis Giannopoulos, Christoph Hölscher, and Iva Barisic. 2016. The Interplay of Pedestrian Navigation, Wayfinding Devices, and Environmental Features in Indoor Settings. In Proceedings of the Symposium on Eye Tracking Research \& Applications. ACM, 85-93.

Pieter Vansteenkiste, Greet Cardon, Eva DâĂŹHondt, Renaat Philippaerts, and M. Lenoir. 2013. The visual control of bicycle steering: The effects of speed and path width. Accident Analysis \& Prevention 51 (2013), 222-227.

Pieter Vansteenkiste, Linus Zeuwts, Greet Cardon, Renaat Philippaerts, and Matthieu Lenoir. 2014. The implications of low quality bicycle paths on gaze behavior of cyclists: A field test. Transportation research part F: traffic psychology and behaviour 23 (2014), 81-87.

Nadir Weibel, Adam Fouse, Colleen Emmenegger, Sara Kimmich, and Edwin Hutchins. 2012. Let's look at the Cockpit: Exploring Mobile Eye-Tracking for Observational Research on the Flight Deck. In Proceedings of the Symposium on Eye Tracking Research \& Applications. ACM, 107-114.

Flora Wenczel, Lisa Hepperle, and Rul von Stülpnagel. 2017. Gaze behavior during incidental and intentional navigation in an outdoor environment. Spatial Cognition \& Computation 17, 1-2 (2017), 121-142.

Giuseppe Angelo Zito, Dario Cazzoli, Loreen Scheffler, Michael Jäger, René Martin Müri, Urs Peter Mosimann, Thomas Nyffeler, FW Mast, and Tobias Nef. 2015. Street crossing behavior in younger and older pedestrians: an eye-and head-tracking study. BMC Geriatrics 15, 1 (2015), 1-10. 